\title{
Whole genomic analysis of a potential recombinant human adenovirus type 1 in Qinghai plateau, China
}

\author{
Juan Yu', Shengcang Zhao ${ }^{2}$ and Huaxiang Rao ${ }^{3^{*}}$
}

\begin{abstract}
Human adenoviruses (HAdVs) are prevalent in patients with respiratory infections, in which recombination has important implications for viral detection and pathogenicity. However, less HAdVs recombination was reported in Qinghai plateau. In this study, we obtained an HAdV-C strain (QH-1665/2018) isolated from an infant aged one month with influenza-like illness in Qinghai Province in 2018. The whole genome sequence was generated by nextgeneration sequencing, and compared with that of other HAdV-C strains available in public. The strain QH-1665/ 2018 genome is comprised of 36,014 nucleotides and encoded 36 putative proteins. Phylogenetic analysis of complete HAdV genomes and 3 major antigen genes (penton, hexon and fiber) showed that strain QH-1665/2018 was clustered into HAdV-1 [P1H1F1]. Recombination analysis based on the RDP4 package and SimPlot software showed that QH-1665/2018 was a recombinant involving HAdV-1, HAdV-2 and HAdV-5, which was then reconfirmed by phylogenetic analysis. Our results suggest that HAdV-C recombination is highly complex, should be focused on, and the epidemiological and virological surveillance should be strengthened in Qinghai Province.
\end{abstract}

Keywords: Human adenovirus (HAdVs), Whole genome sequencing, Recombination analysis

\section{Introduction}

Adenovirus is a non-enveloped, double-stranded DNA virus belonging to the Adenoviridae family, that can cause infections related to the respiratory tract, gastrointestinal tract and eyes in humans [1]. Although HAdV infection is mild and self-limited in the healthy individuals, lifethreatening disease can occur in immune-compromised patients [2, 3]. Currently, human adenoviruses (HAdVs) are classified into seven groups (HAdV-A to HAdV-G), including 52 serotypes and 90 genotypes [4]. Among which, HAdV-C contains HAdV-1, HAdV-2, HAdV-5, HAdV-6, HAdV-57 and HAdV-89, which are common etiological factors in respiratory infections, especially for severe bronchiolitis or pneumonia in children $[5,6]$.

\footnotetext{
* Correspondence: raohuaxiang2006006@163.com

${ }^{3}$ Department of Public Health and Preventive Medicine, Changzhi Medical College, 161 Jiefang East Street, Changzhi 046000, China

Full list of author information is available at the end of the article
}

Complete genomic data have enhanced the understanding of HAdV epidemiology and is an important way to recognize the recombination in HAdV pathogens. In recent years, genetic recombinant of HAdV-C has also been sporadically identified in humans $[4,7,8]$. However, until now, little data on HAdV recombination have been reported in Qinghai Province. Here, we describe the characterization of an HAdV-1 recombinant (QH-1665/2018) isolated from an infant aged one month with influenza-like illness in Qinghai Province in 2018. This information would enhance the understanding of recombination of HAdV-C, and might assist with effective prevention and control of respiratory adenovirus infection in Qinghai Province.

\section{Materials and methods}

\section{Sample collection and identification}

The Qinghai adenovirus strain(QH-1665/2018) was isolated from an outpatient, whom was one month old and 
diagnosed with an influenza-like illness at Women's and Children's Hospital of Qinghai Province on November 26, 2018. Nasopharyngeal swab specimen of this patient was HAdV positive as detected using our previous methods [9].

\section{Virus isolation}

The HAdV positive samples were inoculated onto human laryngeal epidermoid cancer cells (HEp-2) cultured with DMEM containing 2\% FBS for virus isolation. After incubation for 7 days, if no cytopathic effect (CPE) appeared, the cultures were collected and two additional passages were conducted; if CPE appeared, the cultures were passaged again to confirm the presence of virus. QH-1665/ 2018 caused adenovirus-like CPE of HEp- 2 cells, and the cultures underwent three passages to obtain high-titer stocks. The virus-infected cells and supernatant were then collected and used for subsequent genome sequencing.

\section{Whole-genome sequencing}

Whole-genome sequencing of HAdV strain QH-1665/ 2018 was performed on Illumina HiSeq Xten platform (PE 150) by BioGerm (Shanghai, China), and the complete genome of HAdV was then assembled by SPAdes software.

\section{Phylogenetic analysis}

The HAdV nucleotide sequences were analyzed by using BioEdit version 7.0.4.1 and NCBI BLAST software (http://blast.ncbi.nlm.nih.gov/). MEGA 6.06 software was used for phylogenetic analysis of aligned sequences. The phylogenetic tree was generated using the Maximum Likelihood (ML) algorithm. The credibility of the phylogenetic tree was tested by applying a bootstrap test with 1000 replications [10].

\section{Recombination analysis}

The Recombination Detection Program (RDP) package Beta 4.100 was used for identification of recombinant sequences in default mode. A recombination event with a significance of $p<0.01$ in at least three out of seven selected algorithms: RDP, GENECONV, BootScan, Maxchi, Chimaera, SiScan, and 3Seq, was considered to be reliable. Recombination events were then confirmed and visualized with SimPlot Version 3.5.1. Bootscan analysis was used to test potential
A

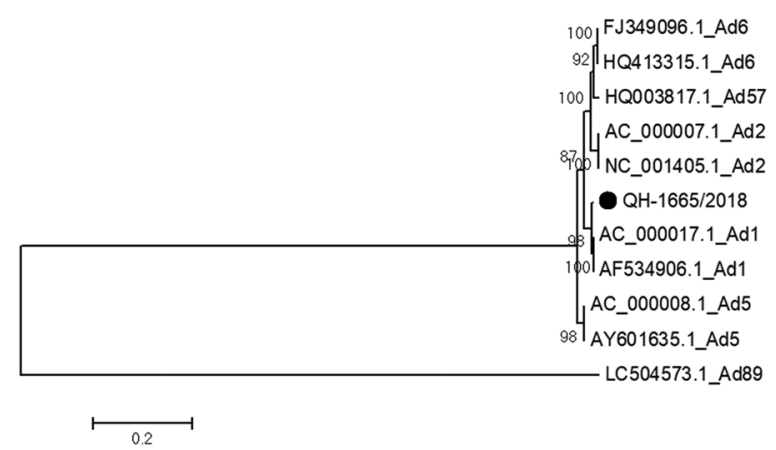

C

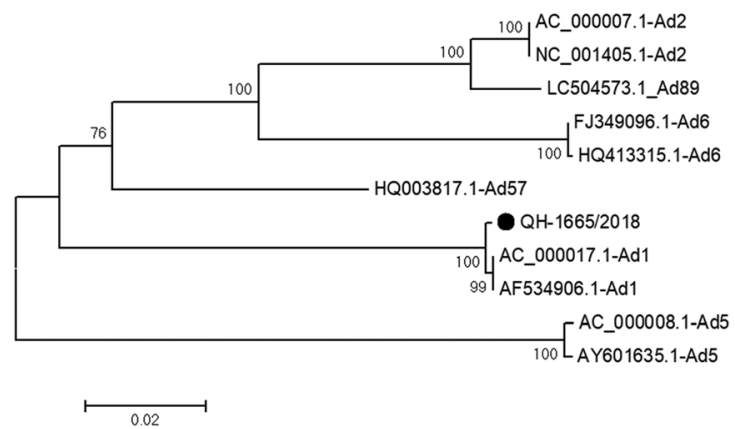

B

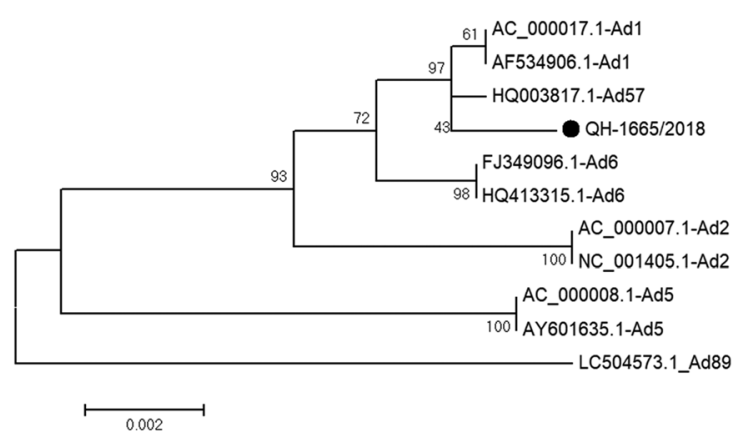

D

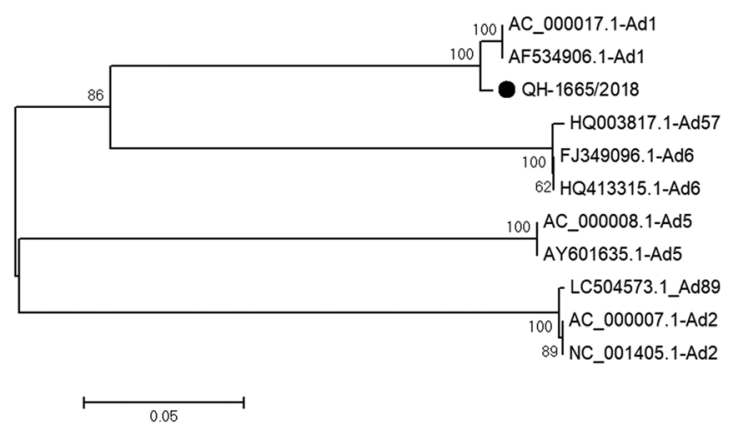

Fig. 1 Phylogenetic analysis of HAdVs based on the complete genome (a), penton (b), hexon (c) and fiber gene (d). The trees were constructed using the Maximum Likelihood (ML) method of MEGA 6.06 with 1000 bootstrap trials. QH-1665/2018 highlighted with a black dot was characterized in this study 
recombination events in the default settings. The sequence of the QH-1665/2018 strain was used as the query sequence and compared to those of other HAdV-C isolates with a sliding window of $200 \mathrm{bp}$.

\section{Results}

\section{Complete genomic characterization}

Using next-generation sequencing, the full-length genomic sequence of the Qinghai HAdV isolate (QH-1665/ 2018) was determined and deposited in GenBank (accession number MN737436). The genome is comprised of 36,014 nucleotides with a GC content of $55.34 \%$ and encodes 36 putative proteins. Whole genome phylogenetic analysis of complete HAdV genomes illustrated that strain QH-1665/2018 was clustered into HAdV-1, and phylogenetic analysis of penton, hexon and fiber genes showed that the 3 major antigen genes were classified into P1, H1, and F1 (Fig. 1).

\section{Homology analysis}

Comparison with the complete genome sequences of the six HAdV-C strains (HAdV-1, HAdV-2, HAdV-5,
HAdV-6, HAdV-57 and HAdV-89) showed that QH$1665 / 2018$ was conserved, sharing the highest nucleotide identity (99.39\%) with HAdV-1. According to Zhang's method [4], we compared the nucleotide sequence identity of coding regions within QH-1665/ 2018 with that of HAdV-C representative strains. The nucleotide sequences of the penton, hexon and fiber genes showed the highest nucleotide identity with HAdV-1, which was consistent with that of the phylogenetic analysis. Furthermore, E1A, pIX, Iva2, DNA polymerase, pIIIa, V, pX, pVI, DBP, $100 \mathrm{~K}$, pVIII, E3 and fiber showed the highest sequence similarity with HAdV-1. Interestingly, we found that the $52 \mathrm{~K}$ gene showed the highest sequence similarity with HAdV-2, the E4 gene showed the highest sequence similarity with HAdV-5, the pTP and protease gene showed the highest sequence similarity with HAdV-6, the E1B region showed the highest sequence similarity with HAdV-57, and the pVII gene showed the highest similarity with both HAdV-6 and HAdV-57. These results suggested that QH-1665/2018 might be a recombinant strain (Table 1).

Table 1 Nucleotide sequence identities between QH-1665/2018 and HAdV-C representative strains

\begin{tabular}{|c|c|c|c|c|c|c|}
\hline \multirow[t]{3}{*}{ Region } & \multicolumn{6}{|c|}{ Nucleotide identity (\%) } \\
\hline & \multicolumn{6}{|c|}{$\mathrm{QH}-1665 / 2018$} \\
\hline & HAdV-C1 & HAdV-C2 & HAdV-C5 & HAdV-C6 & HAdV-C57 & HAdV-C89 \\
\hline E1A & 99.43 & 98.74 & 99.08 & 98.74 & 98.62 & 98.74 \\
\hline E1B & 99.06 & 99.24 & 97.69 & 98.18 & 99.28 & 98.24 \\
\hline plX & 100.00 & 99.53 & 99.76 & 99.53 & 100.00 & 99.53 \\
\hline Iva2 & 99.18 & 98.73 & 98.80 & 98.73 & 99.10 & 98.80 \\
\hline DNA polymearse & 99.28 & 98.86 & 98.72 & 98.89 & 99.16 & 98.80 \\
\hline pTP & 99.50 & 99.50 & 99.10 & 99.55 & 99.35 & 99.35 \\
\hline $52 \mathrm{~K}$ & 99.36 & 99.84 & 98.56 & 99.68 & 99.36 & 99.68 \\
\hline pllla & 99.55 & 99.55 & 99.09 & 99.32 & 99.37 & 99.49 \\
\hline penton & 99.59 & 98.44 & 97.74 & 99.36 & 99.59 & 97.62 \\
\hline $\mathrm{pV} \|$ & 99.50 & 98.66 & 99.00 & 99.83 & 99.83 & 99.16 \\
\hline V & 99.82 & 98.38 & 98.01 & 98.83 & 99.46 & 98.83 \\
\hline $\mathrm{pX}$ & 100.00 & 100.00 & 100.00 & 100.00 & 99.59 & 100.00 \\
\hline $\mathrm{pVl}$ & 99.60 & 97.60 & 97.08 & 96.68 & 96.40 & 99.07 \\
\hline hexon & 99.79 & 88.63 & 85.91 & 84.92 & 88.50 & 88.23 \\
\hline protease & 99.19 & 98.05 & 97.40 & 99.51 & 97.56 & 98.05 \\
\hline DBP & 99.69 & 97.11 & 96.67 & 96.98 & 96.86 & 97.61 \\
\hline $100 \mathrm{~K}$ & 99.63 & 98.15 & 97.40 & 98.35 & 97.65 & 98.85 \\
\hline pVIII & 99.71 & 98.25 & 95.77 & 98.10 & 97.37 & 98.10 \\
\hline E3 & 99.36 & 82.95 & 84.41 & 82.97 & 82.97 & 82.88 \\
\hline fiber & 99.06 & 74.28 & 76.39 & 85.44 & 85.31 & 74.39 \\
\hline E4 & 99.08 & 99.20 & 99.50 & 98.93 & 98.66 & 98.24 \\
\hline Complete genome & 99.39 & 98.24 & 98.43 & 96.99 & 97.37 & 98.26 \\
\hline
\end{tabular}


Table 2 Algorithms of the RDP4 package used to predict the recombination event

\begin{tabular}{|c|c|c|c|c|c|c|c|c|c|}
\hline \multirow{2}{*}{$\begin{array}{l}\text { Recombinant } \\
\text { strain }\end{array}$} & \multirow[t]{2}{*}{ Parent major/minor } & \multirow{2}{*}{$\begin{array}{l}\text { Recombinant } \\
\text { region in } \\
\text { alignment }\end{array}$} & \multicolumn{7}{|c|}{ Model (average $\boldsymbol{p}$-value) } \\
\hline & & & $\overline{\mathrm{RDP}}$ & GENECONV & BootScan & MaxChi & Chimaera & SiScan & $3 \mathrm{Seq}$ \\
\hline \multirow[t]{2}{*}{$\mathrm{QH}-1665 / 2018$} & AC_000017_Ad1/AC_000007_Ad2 & $7655-13,405$ & $2.45 \mathrm{E}-19$ & 4.98E-06 & 1.44E-02 & 0.009607 & $1.37 \mathrm{E}-03$ & 1.03E-02 & $4.08 \mathrm{E}-07$ \\
\hline & AC_000017_Ad1/ AC_000008_Ad5 & $33,039-35,114$ & $2.90 \mathrm{E}-19$ & 3.79E-11 & 3.36E-08 & 5.00E-07 & $6.39 \mathrm{E}-05$ & 2.09E-03 & $8.46 \mathrm{E}-10$ \\
\hline
\end{tabular}

\section{A}

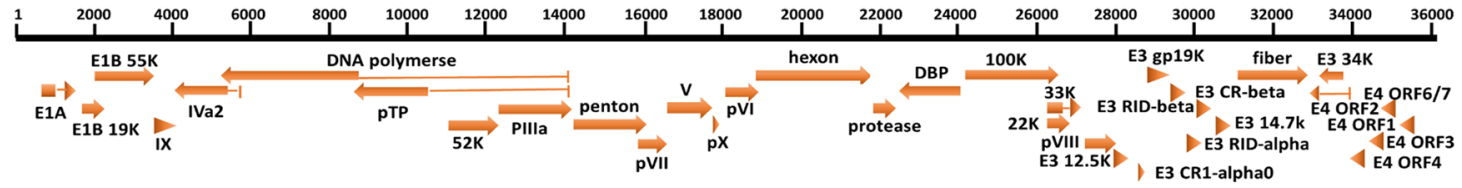

B

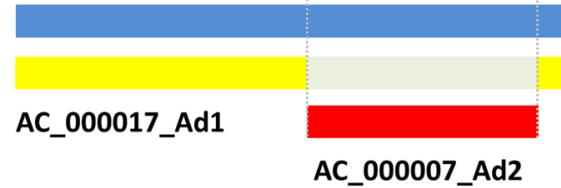

\section{C}

BootScan-Query: QH-1665/2018

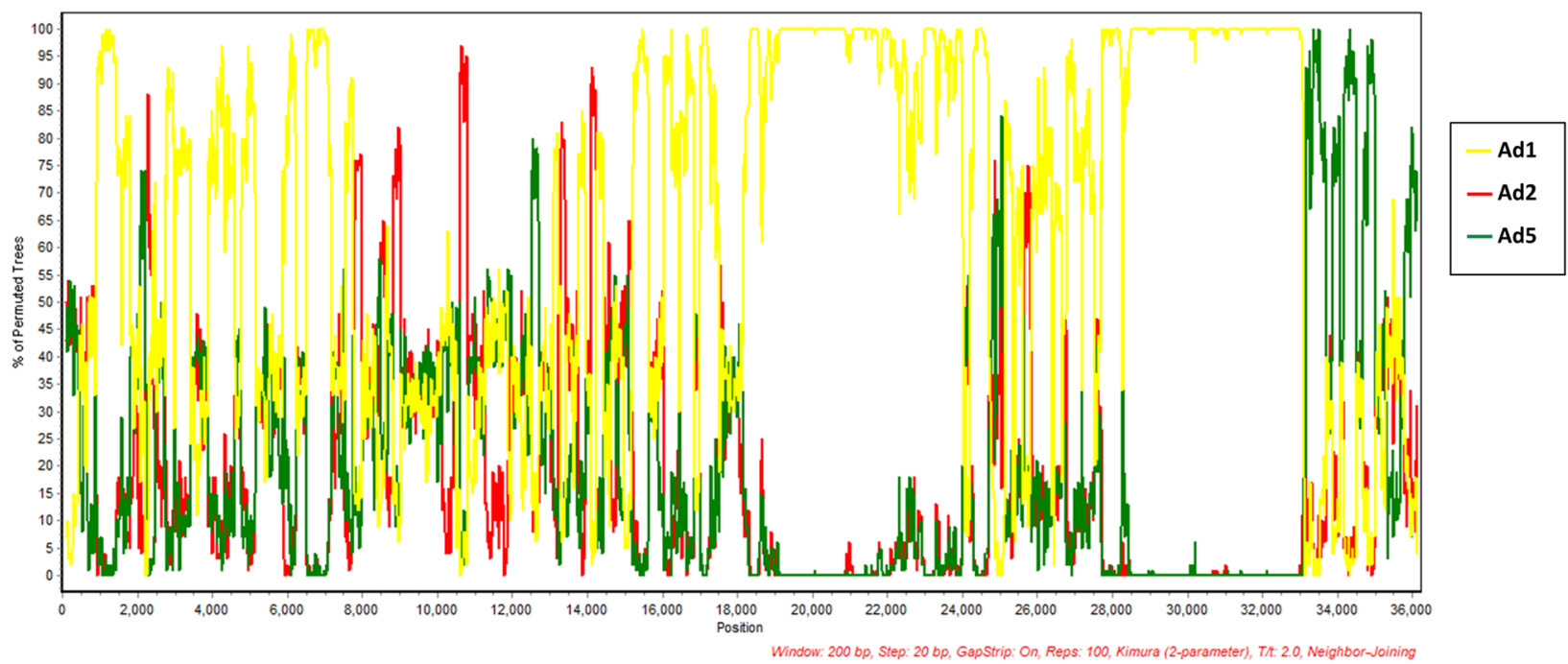

Fig. 2 Genomic recombinant analyses of the complete genome of QH-1665/2018. a Genetic organization of QH-1665/2018. b Recombination events predicted in strain QH-1665/2018. QH-1665/2018 genome is shown with blue. The likely backbone is shown with yellow. Recombination event predicted by RDP4 are shown as red and green, respectively. Likely breakpoint positions are shown above the genome. c BootScan analysis of QH-1665/2018. QH-1665/2018 was used as the query sequence and compared with 3 representative strains of HAdV-C, including HAdV-1 (AC_000017.1), HAdV-2 (AC_000007.1) and HAdV-5 (AC_000008.1). The default setting of SimPlot software was used as follows: window size 200 bp, step size 20 bp. 100 replicates used, gap stripping, distance model (Kimura) and tree model (neighbor-joining) 


\section{Recombination analysis}

To identify the recombination events within the genome of QH-1665/2018, recombination analysis was performed using the RDP4 package with multiple algorithms. Seven algorithms (RDP, GENECONV, BootScan, MaxChi, Chimaera, SiScan, 3Seq) were utilized to predict potential recombination events between the input sequences. The results indicated that it was a highly probable homologous recombinant resulting from HAdV-1 (AC_000017.1), HAdV-2 (AC_000007.1) and HAdV-5 (AC_000008.1) (Table 2). One recombinant event appeared with a beginning breakpoint at around 7648 (without gaps) and an ending breakpoint at around 13,390 (without gaps), with the major parent strain of HAdV-1 and a minor parent strain of HAdV-2, encompassing the genes pTP and $52 \mathrm{k}$ as well as pIIIa partially. Another recombinant event appeared with a beginning breakpoint at around 32,843 (without gaps) and an ending breakpoint at around 34,917 (without gaps), with the major parent strain of HAdV-1 and a minor parent strain of HAdV-5, including most of E4 gene (Fig. 2a, b). BootScan analysis was then performed to confirm the recombination events within the genome of QH-1665/ 2018 by using SimPlot software (Fig. 2c).
The phylogenetic analysis also showed that recombinant region 1 (7648-13,390, pTP, $52 \mathrm{k}$ and partial pIIIa) was clustered in HAdV-2, and recombinant region 2 (32843-34,917, E4) was clustered in HAdV-5, which coincided with previous recombination analysis (Fig. 3). The results re-confirmed that QH-1665/2018 was a recombinant containing HAdV-1, HAdV-2 and HAdV-5 sequences.

\section{Discussion}

Recombination is common and important for the evolution of adenoviruses, which can drive the production of new adenoviruses genotypes [11]. HAdV-B and HAdV-C are the epidemic strains, causing respiratory infections in China [9, 12, 13]. However, few observations on HAdV-C recombinants have been reported compared to HAdV-B [14, 15]. Recently, new adenovirus genotypes have been increasingly recognized based on whole genome sequencing $[15,16]$. The previous studies showed that three recombinant HAdV-C strains (BJ04, BJ09 and CBJ113) have been identified, but with different recombination events $[7,17]$, which indicated that HAdV-C recombination was very complex.
A

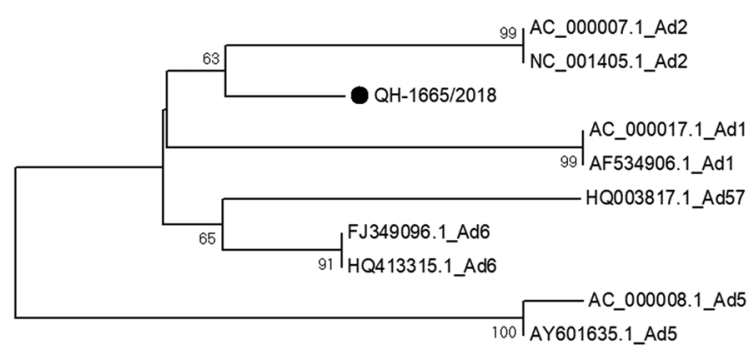

0.001

C

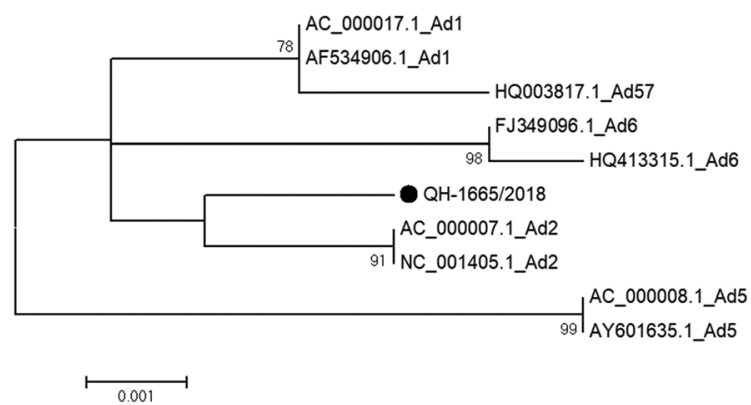

B

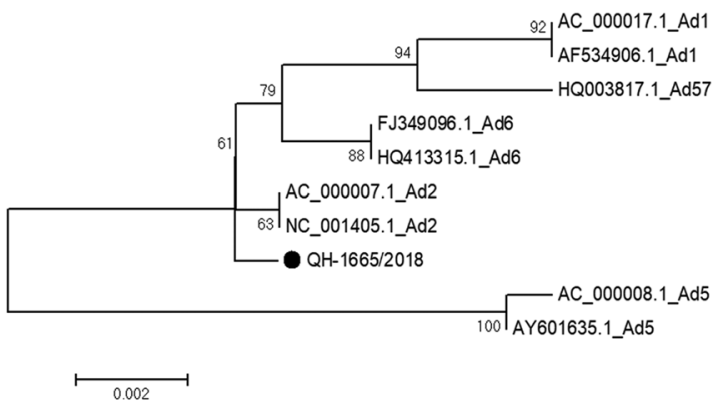

D

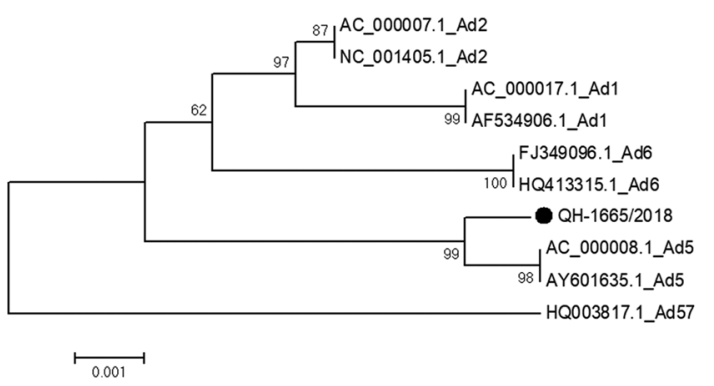

Fig. 3 Phylogenetic analysis based on the recombinant region of QH-1665/2018. a pTP gene. b $52 \mathrm{~K}$ gene. c Partial pllla gene. D: E4 gene. The trees were constructed using the Maximum Likelihood (ML) method of MEGA 6.06 with 1000 bootstraps. QH-1665/2018 highlighted with a black dot was characterized in this study 
In this study, through phylogenetic analysis of HAdV based on the complete genome and 3 major antigen genes (penton, hexon and fiber), QH-1665/2018 was clustered to HAdV-1 [P1H1F1], which could be considered the backbone of the prototype HAdV-1 genome. The comparative genome analysis between QH-1665/ 2018 and other HAdV-C strains displayed several genes that did not show the highest sequence similarity with HAdV-1, for example, $52 \mathrm{~K}$ showed the highest sequence similarity with HAdV-2 and E4 showed the highest sequence similarity with $\mathrm{HAdV}-5$, which indicated $\mathrm{QH}-$ $1665 / 2018$ might be a recombinant. In addition, recombination analysis based on the RDP4 package and SimPlot software both showed that QH-1665/2018 had recombinant events involving HAdV-1, HAdV-2 and HAdV-5, which was re-confirmed by phylogenetic analysis. The recombination areas were located between 7648 and 13,390, which included pTP, $52 \mathrm{k}$ and partially pIIIa, and between 32,843 and 34,917, which included most of E4. Interestingly, the recombination event occurred at pTP, $52 \mathrm{k}$, pIIIa and E4 of HAdV-1, but not in known recombination hotspots, such as the penton base, hexon, and fiber [18]. pTP and E4 are early genes, which are associated with viral DNA replication and transcription, and $52 \mathrm{k}$ and pIIIa are late genes, which are associated with viral capsid formation [19, 20]. The function of the recombination events at these loci in HAdV-C evolution remains to be elucidated.

The recombination usually occurs between strains of the same species, and several adenovirus prototype strains were found to be intratypic recombinants [21]. Our previous study showed that HAdV-C (HAdV-1, HAdV-2, HAdV-5, HAdV-6) strains were circulating in Qinghai Province simultaneously [9], which could have provided the opportunity for intratypic recombinant events. It indicated that the recombinant HAdV-C types might have been circulating in Qinghai Province for a long time, but more sequences will be needed for further confirmation. It was reported that HAdV-1 and HAdV-2 could cause a higher morbidity rate than HAdV-5 and HAdV-6 did [7]. Whether recombination would influence the virulence, pathogenicity and clinical characteristics of HAdV strains should be investigated in the future.

\section{Conclusion}

We showed that QH-1665/2018 was a recombinant HAdV-C strain, it arose through the recombination of three HAdV genotypes-HAdV-1, HAdV-2 and HAdV-5. Our results suggest that HAdV-C recombinant might be circulating in Qinghai Province, but large-scale molecular epidemiological investigation of $\mathrm{HAdV}-\mathrm{C}$ recombination is needed to confirm this. Corresponding prevention and control strategies should be taken into consideration in future work.

\section{Abbreviations}

CPE: Cytopathic effect; HAdVs: Human adenovirus; HEp-2: Human laryngeal epidermoid cancer cells; ML: Maximum Likelihood;

RDP: Recombination Detection Program

\section{Acknowledgements}

We wish to express our gratitude to the staff, who participated in the nasopharyngeal swab specimens collection.

\section{Authors' contributions}

JY and HXR conceived and designed the study. JY performed the experiments, analyzed the data and wrote the manuscript. SCZ supplied the clinical specimens. The author(s) read and approved the final manuscript.

\section{Funding}

The present work was supported by Doctor research startup foundation of Changzhi Medical College (BS201912, BS201921), Key Project of Qinghai Health and Family Planning Commission (2017-wjzd-08) and Qinghai

Thousand People Plan.

\section{Availability of data and materials}

The data supporting the conclusions of this article are included within the article.

\section{Ethics approval and consent to participate}

This study was approved by the Ethics Committee of Qinghai Center for Disease Control and Prevention, the related procedures were performed according to the guideline of National Influenza Surveillance Program (Edition 2017). The specimen was collected from nostril, posterior pharyngeal wall and bilateral tonsils by using sterile swabs gently, which was safety and non-invasive. Before the sample collection, all the subjects or their guardian were informed the purpose of this study, and signed the informed consent.

\section{Consent for publication}

Not applicable.

\section{Competing interests}

The authors declare no competing interests.

\section{Author details}

${ }^{1}$ Department of Basic Medical Sciences, Changzhi Medical College, 161 Jiefang East Street, Changzhi 046000, China. ${ }^{2}$ Center of Hygiene Inspection, Qinghai Center for Disease Control and Prevention, 55 Bayi Middle Road, Xining 810007, China. ${ }^{3}$ Department of Public Health and Preventive Medicine, Changzhi Medical College, 161 Jiefang East Street, Changzhi 046000, China.

Received: 26 March 2020 Accepted: 15 July 2020

Published online: 22 July 2020

\section{References}

1. Ferreyra $L$, Giordano MO, Martinez LC, Barril PA, Masachessi G, Isa MB, Poma R, Rajal V, Biganzoli P, Nates SV, et al. Tracking novel adenovirus in environmental and human clinical samples: no evidence of endemic human adenovirus type 58 circulation in Cordoba city, Argentina. Epidemiol Infect. 2015;143:1427-31.

2. Echavarria M. Adenoviruses in immunocompromised hosts. Clin Microbiol Rev. 2008;21:704-15.

3. Lion T. Adenovirus infections in immunocompetent and immunocompromised patients. Clin Microbiol Rev. 2014:27:441-62.

4. Zhang W, Huang L. Genome analysis of a novel recombinant human adenovirus type 1 in China. Sci Rep. 2019:9:4298.

5. Binder AM, Biggs HM, Haynes AK, Chommanard C, Lu X, Erdman DD, Watson JT, Gerber SI. Human adenovirus surveillance - United States, 20032016. MMWR Morb Mortal Wkly Rep. 2017;66:1039-42.

6. Takahashi K, Gonzalez G, Kobayashi M, Hanaoka N, Carr MJ, Konagaya M, Nojiri N, Ogi M, Fujimoto T. Pediatric infections by human mastadenovirus C 
types 2, 89, and a recombinant type detected in Japan between 2011 and 2018. Viruses. 2019;11:1131.

7. Wang Y, Li Y, Lu R, Zhao Y, Xie Z, Shen J, Tan W. Phylogenetic evidence for intratypic recombinant events in a novel human adenovirus $C$ that causes severe acute respiratory infection in children. Sci Rep. 2016;6:23014.

8. Walsh MP, Seto J, Liu EB, Dehghan S, Hudson NR, Lukashev AN, Ivanova O, Chodosh J, Dyer DW, Jones MS, et al. Computational analysis of two species C human adenoviruses provides evidence of a novel virus. J Clin Microbiol. 2011:49:3482-90.

9. Yu J, Li H, Lu NN, Lei YJ, Jiang SY, Zhao SC, Rao HX. Molecular characterization of human respiratory adenovirus infection in children from November 2016 to October 2017 in Xining City, China. Biomed Environ Sci. 2019:32:38-41.

10. Sriwanna P, Chieochansin T, Vuthitanachot C, Vuthitanachot V, Theamboonlers A, Poovorawan Y. Molecular characterization of human adenovirus infection in Thailand, 2009-2012. Virol J. 2013;10:193.

11. Walsh MP, Chintakuntlawar A, Robinson CM, Madisch I, Harrach B, Hudson NR, Schnurr D, Heim A, Chodosh J, Seto D, et al. Evidence of molecular evolution driven by recombination events influencing tropism in a novel human adenovirus that causes epidemic keratoconjunctivitis. PLoS One. 2009;: :e5635.

12. Li Y, Zhou W, Zhao Y, Wang Y, Xie Z, Lou Y, Tan W. Molecular typing and epidemiology profiles of human adenovirus infection among paediatric patients with severe acute respiratory infection in China. PLoS One. 2015;10; e0123234.

13. Chen M, Zhu Z, Huang F, Liu D, Zhang T, Ying D, Wu J, Xu W. Adenoviruses associated with acute respiratory diseases reported in Beijing from 2011 to 2013. PLoS One. 2015;10:e121375.

14. Wang W, Liu Y, Zhou Y, Gu L, Zhang L, Zhang X, Chen M, Zou Z, Qiu W, Hu $X$, et al. Whole-genome analyses of human adenovirus type 55 emerged in Tibet, Sichuan and Yunnan in China, in 2016. PLoS One. 2017;12:e0189625.

15. Ismail AM, Cui T, Dommaraju K, Singh G, Dehghan S, Seto J, Shrivastava S, Fedorova NB, Gupta N, Stockwell TB, et al. Genomic analysis of a large set of currently-and historically-important human adenovirus pathogens. Emerg Microbes Infect. 2018;7:10

16. Yang Z, Zhu Z, Tang L, Wang L, Tan X, Yu P, Zhang Y, Tian X, Wang J, Li D, et al. Genomic analyses of recombinant adenovirus type 11a in China. J Clin Microbiol. 2009;47:3082-90

17. Mao N, Zhu Z, Rivailler P, Chen M, Fan Q, Huang F, Xu W. Whole genomic analysis of two potential recombinant strains within human mastadenovirus species C previously found in Beijing, China. Sci Rep. 2017;7:15380.

18. Gonzalez G, Koyanagi KO, Aoki K, Watanabe H. Interregional coevolution analysis revealing functional and structural interrelatedness between different genomic regions in human Mastadenovirus D. J Virol. 2015;89: 6209-17.

19. Russell WC. Adenoviruses: update on structure and function. J Gen Virol. 2009;90:1-20,

20. Lauer KP, Llorente I, Blair E, Seto J, Krasnov V, Purkayastha A, Ditty SE, Hadfield TL, Buck C, Tibbetts C, et al. Natural variation among human adenoviruses: genome sequence and annotation of human adenovirus serotype 1. J Gen Virol. 2004;85:2615-25.

21. Lukashev AN, Ivanova OE, Eremeeva TP, Iggo RD. Evidence of frequent recombination among human adenoviruses. J Gen Virol. 2008;89:380-8.

\section{Publisher's Note}

Springer Nature remains neutral with regard to jurisdictional claims in published maps and institutional affiliations.

Ready to submit your research? Choose BMC and benefit from:
- fast, convenient online submission
- thorough peer review by experienced researchers in your field
- rapid publication on acceptance
- support for research data, including large and complex data types
- gold Open Access which fosters wider collaboration and increased citations
- maximum visibility for your research: over 100M website views per year
At BMC, research is always in progress.
Learn more biomedcentral.com/submissions

